\title{
Optimal Pairs Trading: A Stochastic Control Approach
}

\author{
Supakorn Mudchanatongsuk, James A. Primbs and Wilfred Wong
}

\begin{abstract}
In this paper, we propose a stochastic control approach to the problem of pairs trading. We model the logrelationship between a pair of stock prices as an OrnsteinUhlenbeck process and use this to formulate a portfolio optimization based stochastic control problem. We are able to obtain the optimal solution to this control problem in closed form via the corresponding Hamilton-Jacobi-Bellman equation. We also provide closed form maximum-likelihood estimation values for the parameters in the model. The approach is illustrated with a numerical example involving simulated data for a pair of stocks.
\end{abstract}

\section{INTRODUCTION}

Pairs trading is an investment strategy based on identifying pairs of stocks that typically trade in a predictable relation to one another. When deviations to this predictable relationship occur, a pairs trading strategy will trade in order to profit from a return to the typical relationship. The standard example is a pair of highly correlated stocks in the same industry, such as Coca-Cola and Pepsi or Wal-Mart and Target. The stocks in the pair tend to trade relative to one another. For example, the overall market value of WalMart and Target tend to move together (since they are in the same industry), and Wal-Mart's market value is roughly 3 to 4 times that of Target (due to the size difference between the two companies). When deviations to this basic relationship occur, such as Wal-Mart trading for 5 times Target, a pairs trading strategy would bet on a return to the standard relationship by purchasing Target and selling and equal dollar amount of Wal-Mart. Thus, a pairs trading strategy bets solely on the relationship between the pair of stocks and not on the overall movement of the industry or market that they are in.

It is well known that pairs trading is a common strategy among many hedge funds. However, there is not a significant amount of academic literature devoted to it due to its proprietary nature. For a review of some of the existing academic models, see [1], [3], [2].

In our paper, we develop a model whereby the difference in the log-price of a pair of stocks (known as the spread) is an Ornstein-Uhlenbeck process [9]. We then use a stochastic control formulation of a dynamic portfolio optimization problem where one may either trade based on the spread (by buying and selling equal amounts of the stocks in the pair) or place money in a risk free asset. Using an ansatz motivated by [5] and [4], we are able to find a closed form solution to the control problem under a power utility on terminal wealth.

The authors are with the Department of Management Science and Engineering, Stanford University, Stanford, CA 94022, USA supakornestanford.edu, japrimbsestanford.edu, wilfredwestanford.edu
Furthermore, we show that parameter values for the model may be obtained in closed form under maximum likelihood estimation. Thus, we obtain a simple and practical solution to the modeling and optimal trading of pairs.

The paper is organized as follows. In Section II, we formulate the pairs trading problem as a stochastic optimal control problem. In Section III, we solve the corresponding Hamilton-Jacobi-Bellman equation in closed form. Since the focus of this paper is the application of stochastic control to pairs trading, we do not provide a rigorous verification theorem for this HJB solution. For readers who are interested in this topic, refer to [5]. In Section IV, we provide a numerical study involving simulated data. Section $\mathrm{V}$ gives conclusions. Finally, the maximum likelihood estimates for parameter values in closed form are provided in the Appendix.

\section{PROBLEM FORMULATION}

This section presents the basic asset price models and formulates a pairs trading stochastic control problem. We begin by describing the asset, spread, and wealth dynamics.

\section{A. Asset, Spread and Wealth Dynamics}

We assume that a risk-free asset $M(t)$ exists with a riskfree rate of $r$ compounded continuously. Thus, $M(t)$ satisfies the dynamics

$$
d M(t)=r M(t) d t
$$

Let $A(t)$ and $B(t)$ denote respectively the prices of the pair of stocks $A$ and $B$ at time $t$. We assume that stock $B$ follows a geometric Brownian motion

$$
d B(t)=\mu B(t) d t+\sigma B(t) d Z(t)
$$

where $\mu$ is the drift, $\sigma$ is the volatility, and $Z(t)$ is a standard Brownian motion.

Let $X(t)$ denote the spread of the two stocks at time $t$, defined as

$$
X(t)=\ln (A(t))-\ln (B(t)) .
$$

We assume that the spread follows an Ornstein-Uhlenbeck process

$$
d X(t)=k(\theta-X(t)) d t+\eta d W(t)
$$

where $k(\theta-X(t))$ is the drift term that represents the expected instantaneous change in the spread at time $t$, and $\theta$ is the long-term equilibrium level to which the spread reverts. The rate of reversion is represented by the parameter $k$, 
which has to be positive to ensure stability around the equilibrium value. The standard deviation parameter, $\eta$, determines the volatility of the spread. $W(t)$ is a standard Brownian motion where $\rho$ denotes the instantaneous correlation coefficient between $Z(t)$ and $W(t)$ (i.e. $E[d W(t) d Z(t)]=$ $\rho d t)$.

By using (2), (3), (4) and Ito's lemma [7], we are able to obtain the dynamics of $A(t)$ as

$$
\begin{gathered}
d A(t)=\left(\mu+k(\theta-X(t))+\frac{1}{2} \eta^{2}+\rho \sigma \eta\right) A(t) d t \\
+\sigma A(t) d Z(t)+\eta A(t) d W(t),
\end{gathered}
$$

where the $\rho \sigma \eta$ term comes from the covariance between the Wiener processes $W(t)$ and $Z(t)$.

Let $V(t)$ be the value of a self-financing pairs-trading portfolio and let $h(t)$ and $\tilde{h}(t)$ denote respectively the portfolio weights for stocks $A$ and $B$ at time $t$. Additionally, we only allow ourselves to trade stocks $A$ and $B$ as a pair, (i.e. we are only allowed to go short one of them and long the other in equal dollar amount). Thus, we require that

$$
h(t)=-\tilde{h}(t) .
$$

Finally, noting that the portfolio weight on the risk-free asset is always 1 , the wealth dynamics of the portfolio value is given by

$$
d V(t)=V(t)\left\{h(t) \frac{d A(t)}{A(t)}+\tilde{h}(t) \frac{d B(t)}{B(t)}+\frac{d M(t)}{M(t)}\right\} .
$$

Using (1), (2), (5), and (6), we can rewrite (7) as

$$
\begin{aligned}
d V(t)= & V(t)\left\{\left[h(t)\left(k(\theta-X(t))+\frac{1}{2} \eta^{2}+\rho \sigma \eta\right)\right.\right. \\
& +r] d t+\eta d W(t)\} .
\end{aligned}
$$

\section{B. Formulation as a Stochastic Control Problem}

We formulate the portfolio optimization pair-trading problem as a stochastic optimal control problem. We assume that an investor's preference can be represented by the utility function $U(x)=\frac{1}{\gamma} x^{\gamma}$, with $x \geq 0$ and $\gamma<1$. In this formulation, our objective is to maximize expected utility at the final time $T$. Thus, we seek to solve

$$
\begin{aligned}
\sup _{h(t)} & E\left[\frac{1}{\gamma}(V(T))^{\gamma}\right] \\
\text { subject to: } & V(0)=v_{0}, \quad X(0)=x_{0} \\
d X(t)= & k(\theta-X(t)) d t+\eta d W(t) \\
d V(t)= & V(t)\left(\left(h ( t ) \left(k(\theta-X(t))+\frac{1}{2} \eta^{2}\right.\right.\right. \\
& +\rho \sigma \eta)+r) d t+\eta d W(t)),
\end{aligned}
$$

where the supremum is taken over strategies $h(t)$ that are adapted to the filtration generated by $Z(t)$ and $W(t)$. (For a rigorous formulation in a related setting, see [5].)

In this optimal control problem, the first constraint just specifies the initial wealth of our portfolio and the spread. The second and third constraints describe the spread and wealth dynamics respectively.

In the following section, we show that a closed form solution to the above stochastic control problem exists.

\section{ANALYTICAL SOLUTION}

Let $G(t, v, x)$ denote the value function. By standard arguments, one may show that the Hamilton-Jacobi-Bellman (HJB) equation coresponding to our stochastic control problem is

$$
\begin{aligned}
G_{t}+\sup _{h}\left\{\frac { 1 } { 2 } \left[h^{2} \eta^{2} v^{2} G_{v v}+\eta^{2} G_{x x}\right.\right. & \\
+ & \left.2 h \eta^{2} v G_{v x}\right]+\left[h k(\theta-x)+\frac{1}{2} h \eta^{2}\right. \\
& \left.+h \rho \eta \sigma+r] v G_{v}-k(x-\theta) G_{x}\right\}=0,
\end{aligned}
$$

subject to the terminal condition

$$
G(T, v, x)=v^{\gamma},
$$

where the subscripts on $G$ denote partial derivative.

For notational ease we let $b=-k(x-\theta)+\frac{1}{2} \eta^{2}+\rho \sigma \eta$ and rewrite (9) as

$$
\begin{aligned}
G_{t}+\sup _{h}\left\{\frac { 1 } { 2 } \left[h^{2} \eta^{2} v^{2} G_{v v}+\eta^{2} G_{x x}\right.\right. \\
\left.+2 h \eta^{2} v G_{v x}\right]+[h b+r] v G_{v} \\
\left.+\left[b-\frac{1}{2} \eta^{2}-\rho \sigma \eta\right] G_{x}\right\}=0 .
\end{aligned}
$$

The first order condition for the maximization in (11) is

$$
h^{*} \eta^{2} v G_{v v}+\eta^{2} G_{v x}+b G_{v}=0 .
$$

Assuming $G_{v v}<0$, the first order condition (12) is also sufficient, yielding

$$
h^{*}=-\frac{\eta^{2} G_{v x}+b G_{v}}{\eta^{2} v G_{v v}} .
$$

Plugging (13) back into (11) yields

$$
\begin{gathered}
\eta^{2} G_{t} G_{v v}-\frac{1}{2} \eta^{4} G_{v x}^{2}-\frac{1}{2} b^{2} G_{v}^{2}-b \eta^{2} G_{v} G_{v x} \\
+\frac{1}{2} \eta^{4} G_{v v} G x x+r \eta^{2} v G_{v} G_{v v} \\
-k(x-\theta) \eta^{2} G_{x} G_{v v}=0 .
\end{gathered}
$$

Thus, we must solve the partial differential equation (14) in order to determine an optimal strategy.

\section{A. Closed Form Solution}

To obtain a closed form solution, we consider the following separation ansatz that was motivated by [5] where a different portfolio optimization problem under Vasicek [10] term structure dynamics was solved,

$$
G(t, v, x)=f(t, x) v^{\gamma},
$$

with the condition that

$$
f(T, x)=1 \quad \forall x .
$$

For this choice of ansatz, (14) becomes

$$
\begin{aligned}
& (\gamma-1) \eta^{2} f f_{t}-\frac{1}{2} \gamma \eta^{4} f_{x}^{2}-\frac{1}{2} \gamma b^{2} f^{2}-\frac{1}{2} \gamma \eta^{4} f f_{x} \\
& \quad-\gamma \rho \sigma \eta^{3} f f_{x}+\frac{1}{2}(\gamma-1) \eta^{4} f f_{x x} \\
& \quad+\gamma(\gamma-1) r \eta^{2} f^{2}+k(x-\theta) \eta^{2} f f_{x}=0 .
\end{aligned}
$$

We then use the following ansatz for $f(t, x)$

$$
f(t, x)=g(t) e^{x \beta(t)+x^{2} \alpha(t)} .
$$


Since $f(t, x)$ satisfies the condition (16), the following conditions must be imposed on the ansatz for $f(t, x)$

$$
g(T)=1, \beta(T)=0, \alpha(T)=0 .
$$

With (18), we can rewrite (17) as

$$
\begin{gathered}
\frac{(\gamma-1) \eta^{2} g^{\prime}}{g}-\frac{1}{2} \gamma b^{2}+\left[(\gamma-1) \eta^{2} \alpha^{\prime 4} \alpha^{2}+2 k \eta^{2} \alpha\right] x^{2} \\
+\left[(\gamma-1) \eta^{2} \beta^{\prime 4} \alpha-2 \gamma \rho \sigma \eta^{3} \alpha-2 \eta^{4} \beta \alpha+k \eta^{2} \beta\right. \\
\left.-2 k \theta \eta^{2} \alpha\right] x+\left[-\frac{1}{2} \gamma \eta^{4} \beta-\gamma \rho \sigma \eta^{3} \beta-\frac{1}{2} \eta^{4} \beta^{2}\right. \\
\left.+(\gamma-1) \eta^{4} \alpha+\gamma(\gamma-1) r \eta^{2}-k \theta \eta^{2} \beta\right]=0 .
\end{gathered}
$$

Plugging back $b=-k(x-\theta)+\frac{1}{2} \eta^{2}+\rho \sigma \eta$ into (20) yields

$$
\begin{aligned}
& \frac{(\gamma-1) \eta^{2} g^{\prime}}{g}+\left\{\left[(\gamma-1) \eta^{2}\right] \alpha^{\prime 4}\right] \alpha^{2}+\left[2 k \eta^{2}\right] \alpha \\
& \left.\quad+\left[-\frac{1}{2} \gamma k^{2}\right]\right\} x^{2}+\left\{\left[(\gamma-1) \eta^{2}\right] \beta^{\prime 2}-2 \eta^{4} \alpha\right] \beta \\
& \quad+\left[-\gamma \eta^{4} \alpha-2 \gamma \rho \sigma \eta^{3} \alpha-2 k \theta \eta^{2} \alpha+\gamma k^{2} \theta+\frac{1}{2} \gamma k \eta^{2}\right. \\
& \quad+\gamma k \rho \eta \sigma]\} x+\left\{-\frac{1}{2} \gamma \eta^{4} \beta-\gamma \rho \sigma \eta^{3} \beta-\frac{1}{2} \eta^{4} \beta^{2}\right. \\
& \quad+(\gamma-1) \eta^{4} \alpha+\gamma(\gamma-1) r \eta^{2}-k \theta \eta^{2} \beta-\frac{1}{2} \gamma k^{2} \theta^{2} \\
& \quad-\frac{1}{2} \gamma k \theta \eta^{2}-\gamma k \theta \rho \sigma \eta-\frac{1}{8} \gamma \eta^{4}-\frac{1}{2} \gamma \rho \sigma \eta^{3} \\
& \left.\quad-\frac{1}{2} \gamma \rho^{2} \sigma^{2} \eta^{2}\right\}=0 .
\end{aligned}
$$

Setting the coefficient of $x^{2}$ in (21) to be zero yields an ordinary differential equation for $\alpha(t)$

$$
\left.\left[(\gamma-1) \eta^{2}\right] \alpha^{\prime 4}\right] \alpha^{2}+\left[2 k \eta^{2}\right] \alpha+\left[-\frac{1}{2} \gamma k^{2}\right]=0 .
$$

Similarly, setting the coefficient of $x$ in (21) to be zero yields an ordinary differential equation for $\beta(t)$

$$
\begin{gathered}
\left.\left[(\gamma-1) \eta^{2}\right] \beta^{\prime 2}-2 \eta^{4} \alpha\right] \beta+\left[-\gamma \eta^{4} \alpha-2 \gamma \rho \sigma \eta^{3} \alpha\right. \\
\left.-2 k \theta \eta^{2} \alpha+\gamma k^{2} \theta+\frac{1}{2} \gamma k \eta^{2}+\gamma k \rho \eta \sigma\right]=0 .
\end{gathered}
$$

Finally, if both (21) and (22) hold, then (21) reduces to an ordinary differential equation for $g(t)$

$$
\begin{aligned}
& (\gamma-1) \eta^{2} g^{\prime}+\left[-\frac{1}{2} \gamma \eta^{4} \beta-\gamma \rho \sigma \eta^{3} \beta-\frac{1}{2} \eta^{4} \beta^{2}\right. \\
& \quad+(\gamma-1) \eta^{4} \alpha+\gamma(\gamma-1) r \eta^{2}-k \theta \eta^{2} \beta-\frac{1}{2} \gamma k^{2} \theta^{2} \\
& \quad-\frac{1}{2} \gamma k \theta \eta^{2}-\gamma k \theta \rho \sigma \eta-\frac{1}{8} \gamma \eta^{4}-\frac{1}{2} \gamma \rho \sigma \eta^{3} \\
& \left.\quad-\frac{1}{2} \gamma \rho^{2} \sigma^{2} \eta^{2}\right] g=0 .
\end{aligned}
$$

Noting that (21) is a Riccati equation for $\alpha(t)$, and (22) and (23) are first order linear ordinary differential equations for $\beta(t)$ and $g(t)$, respectively, one may obtain the solution in closed form as,

$$
\begin{aligned}
& \alpha(t)=\frac{k(1-\sqrt{1-\gamma})}{2 \eta^{2}} . \\
& \left\{1+\frac{2 \sqrt{1-\gamma}}{1-\sqrt{1-\gamma}-(1+\sqrt{1-\gamma}) \exp \left(\frac{2 k(T-t)}{\sqrt{1-\gamma}}\right)}\right\},
\end{aligned}
$$

$$
\beta(t)=\frac{1}{2 \eta^{2}\left[(1-\sqrt{1-\gamma})-(1+\sqrt{1-\gamma}) \exp \left(\frac{2 k(T-t)}{\sqrt{1-\gamma}}\right)\right]} .
$$

$$
\begin{aligned}
& \left\{\gamma \sqrt{1-\gamma}\left(\eta^{2}+2 \rho \sigma \eta\right)\left[1-\exp \left(\frac{2 k(T-t)}{\sqrt{1-\gamma}}\right)\right]^{2}\right. \\
& \left.-\gamma\left(\eta^{2}+2 \rho \sigma \eta+2 k \theta\right)\left[1-\exp \left(\frac{2 k(T-t)}{\sqrt{1-\gamma}}\right)\right]\right\}
\end{aligned}
$$

and

$$
g(t)=\exp \left(\frac{-\int_{t}^{T} u(s) d s}{(1-\gamma) \eta^{2}}\right)
$$

where $u$ is given by

$$
\begin{aligned}
u(t)=-\frac{1}{2} \gamma \eta^{4} \beta(t)-\gamma \rho \sigma \eta^{3} \beta(t)-\frac{1}{2} \eta^{4} \beta^{2}(t) \\
+(\gamma-1) \eta^{4} \alpha(t)+\gamma(\gamma-1) r \eta^{2}-k \theta \eta^{2} \beta(t) \\
\quad-\frac{1}{2} \gamma k^{2} \theta^{2}-\frac{1}{2} \gamma k \theta \eta^{2}-\gamma k \theta \rho \sigma \eta-\frac{1}{8} \gamma \eta^{4} \\
\quad-\frac{1}{2} \gamma \rho \sigma \eta^{3}-\frac{1}{2} \gamma \rho^{2} \sigma^{2} \eta^{2} .
\end{aligned}
$$

With (24), (25) and (26), the ansatz $f(t, x)$ can be computed via (18). Then $G(t, v, x)$ can in turn be computed via (15). Consequently, the optimal weight $h^{*}(t)$ can be obtained via (13)

$$
h^{*}(t, x)=\frac{1}{1-\gamma}\left[\beta(t)+2 x \alpha(t)-\frac{k(x-\theta)}{\eta^{2}}+\frac{\rho \sigma}{\eta}+\frac{1}{2}\right] .
$$

With the above closed form solution in hand, in the next section we turn to an illustrative numerical example. The problem of parameter estimation will be discussed in the Appendix.

\section{NUMERICAL RESULTS}

The results of Section III give easily computed expressions for the optimal portfolio strategy in our model of pairs trading. In this section, we test the strategy using simulated data for a pair of stocks. Since the objective of this paper is to develop an optimal pair-trading strategy instead of estimating the corresponding parameters, we hard-code the parameter values when computing the optimal strategy (13). The details are as follows.

As the stock price of $B$ is lognormal (see (34)), we simulate the price process by taking a series of 1-day time periods and then stepping the process forward day by day as in [6] via

$$
\ln \left(B\left(t_{k+1}\right)\right)-\ln \left(B\left(t_{k}\right)\right)=\mu \Delta t+\sigma \epsilon\left(t_{k}\right) \sqrt{\Delta t},
$$

where $t_{k}$ denotes day $k . \Delta t=1 / 251$ (we have assumed 251 trading days in a year) and $\epsilon\left(t_{k}\right)$ 's are normal random variables of mean 0 and standard deviation 1 and $\epsilon\left(t_{i}\right)$ are uncorrelated with $\epsilon\left(t_{j}\right)$ for $i \neq j$. Setting $\mu$ to be 0.3 and $\sigma$ to be 0.1 , the simulated stock price of $B$ is obtained through (29) as

$$
B\left(t_{k+1}\right)=e^{\mu \Delta t+\sigma \epsilon\left(t_{k}\right) \sqrt{\Delta t}} B\left(t_{k}\right) .
$$

Similarly, since the spread $X(t)$ is normally distributed (see (35)), we can simulate the spread process by using the 
method described in [1]

$$
\begin{aligned}
X\left(t_{k+1}\right)= & \theta\left(1-e^{-k \Delta t}\right)+e^{-k \Delta t} X\left(t_{k}\right) \\
& +\sqrt{\frac{\eta^{2}}{2 k}\left(1-e^{-2 k t}\right)} \delta\left(t_{k}\right),
\end{aligned}
$$

where $\Delta t=1 / 251 . \delta\left(t_{k}\right)$ 's are normal random variables of mean 0 and standard deviation 1 and $\delta\left(t_{i}\right)$ are uncorrelated with $\delta\left(t_{j}\right)$ for $i \neq j$. We use $\theta=1, \kappa=5$ and $\eta=0.5$

Furthermore, since we assume that the stock price and the spread are correlated by $\rho$, the covariance of $\epsilon\left(t_{k}\right)$ and $\delta\left(t_{k}\right)$ are set to be $\rho$ for $k=1,2, \ldots$ when generating these two standard normal random variables. We set $\rho$ to be 0.19 .

Finally, from (3), the stock price of $A$ can be obtained as

$$
A\left(t_{k}\right)=B\left(t_{k}\right) e^{X\left(t_{k}\right)} \text {. }
$$

We ran the above simulations for one year (251 trading days) and obtained the stock price paths for $A$ and $B$ in Figure IV.

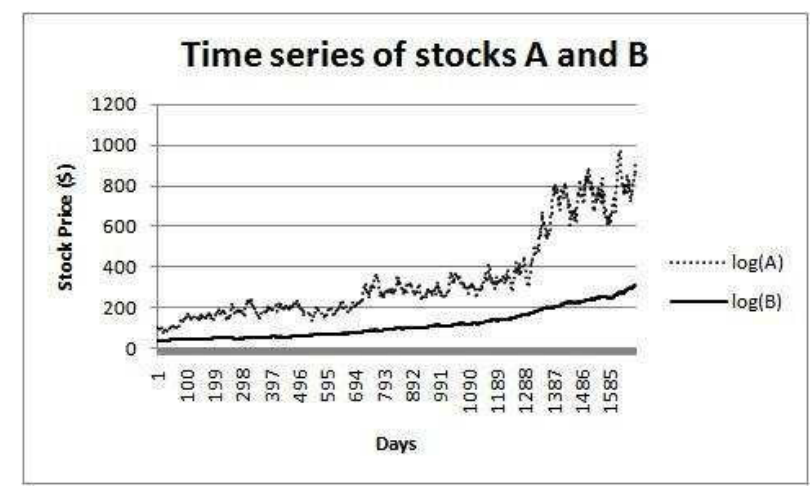

Fig. 1. Time series of the prices of stocks A and B.

With the above simulated data and the corresponding parameter values, the optimal strategy on each day can be computed via (13). Figure 2 shows the resulting wealth dynamics with $\gamma=-100$. For this sample path, the value of the portfolio increases from $\$ 1000$ to $\$ 6693$ in five years, representing a yearly return of over $38 \%$ under continuous compounding.

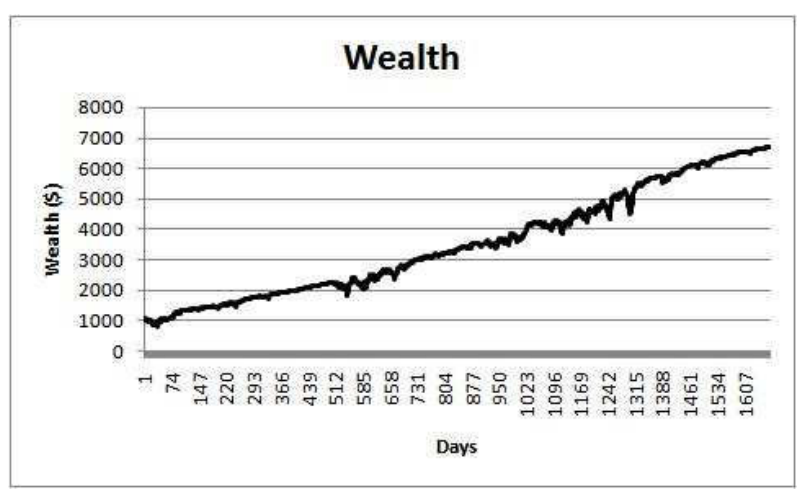

Fig. 2. Wealth dynamics.

Going forward, we are working on using the parameter estimation given in the Appendix to test the strategy on empirical data.

\section{CONCLUSION}

In this paper, we formulated the problem of optimal trading of pairs as a stochastic control problem. We were able to find a closed form solution to this control problem, and derive closed form expressions for maximum likelihood estimators of parameter values. Hence, we obtained an easily implemented approach to the modeling and optimal trading of pairs. This approach was tested on simulated data for a pair of stocks and showed that the strategy performs well.

\section{REFERENCES}

[1] B. Do, R. Faff, and K. Hamza. A new approach to modeling and estimation for pairs trading. In Proceedings of 2006 Financial Management Association European Conference, Stockholm, June 2006.

[2] R. J. Elliott, J. van der Hoek, and W. P. Malcolm. Pairs trading. Quantitative Finance, 5(3):271-276, 2005.

[3] E. Gatev, W. N. Goetzmann, and K. G. Rouwenhorst. Pairs Trading: Performance of a Relative-Value Arbitrage Rule. Review of Financial Studies, 19(3):797-827, 2006.

[4] F. Herzog, G. Dondi, H. Geering, and L. Schumann. Continuous-Time Multivariate Strategic Asset Allocation. Working Paper, 2004.

[5] R. Korn and H. Kraft. A Stochastic Control Approach to Portfolio Problems with Stochastic Interest Rates. SIAM Journal on Control and Optimization, 40(4):1250-1269, 2002.

[6] D.G. Luenberger. Investment Science. Oxford Press, 1998.

[7] B. Oksendal. Stochastic Differential Equations: An Introduction with Applications. Springer, New York, fifth edition, 1998.

[8] J. A. Rice. Mathematical Statistics and Data Analysis. Duxbury Press, third edition, 2006.

[9] G.E. Uhlenbeck and L.S. Ornstein. On the theory of brownian motion. Phys. Rev., 36:823-841, 1930.

[10] O. Vasicek. An Equilibrium Characterization of the Term Structure. Journal of Financial Economics, 5:177-188, 1977.

\section{APPENDIX}

In this section, we present analytic formulas for maximum likelihood parameter value estimators. We consider the problem of using historical data to estimate the parameters $k, \theta$, $\eta, \mu, \sigma$ and $\rho$. First, we let $S(t)=\ln (B(t))$ and $v=\mu-\frac{1}{2} \sigma^{2}$. Applying Ito's lemma to (2) yields

$$
d S(t)=v d t+\sigma d Z(t) .
$$

It can be verified that $S(t)$ is normally distributed

$$
S(t) \sim N\left(S(0)+v t, \sigma^{2} t\right) .
$$

It can also be verified that the spread $X(t)$ is normally distributed

$$
X(t) \sim N\left(X(0) e^{-k t}+\theta\left(1-e^{-k t}\right), \frac{\eta^{2}}{2 k}\left(1-e^{-2 k t}\right)\right),
$$

and that the covariance between $X(t)$ and $S(t)$ is

$$
\operatorname{Cov}(X(t), S(t))=\frac{\rho \eta \sigma}{k}\left(1-e^{k t}\right) .
$$

Let $y(t)=[X(t), S(t)]^{T}$ and suppose that $N+1$ periods of historical data are available, i.e. we have observed $y(t)$ for $t=0,1, \ldots, N$ where $\Delta t$ is the time period between observations.

The likelihood function is given by [8]

$$
f(y(0), \ldots, y(N) \mid k, \theta, \eta, \mu, \sigma, \rho),
$$


where $f(\cdot \mid \cdot)$ is the joint density of the observations given the parameter values. Since $y(t)$ is Markov, the likelihood function can be expressed as (where we have suppressed the parameter values for notational simplicity)

$$
\text { likelihood }=\prod_{t=0}^{T-1} f(y(t+1) \mid y(t))
$$

where $f(y(t+1) \mid y(t))$ is equal to

$$
\begin{gathered}
\frac{1}{2 \pi \sqrt{\operatorname{det}(\Sigma)}} \exp \left\{-\frac{1}{2}(y(t+1)-E[y(t+1) \mid y(t)])^{T} \Sigma^{-1}\right. \\
(y(t+1)-E[y(t+1) \mid y(t)])\}
\end{gathered}
$$

with

$$
\begin{gathered}
E[y(t+1) \mid y(t)]=\left[\begin{array}{c}
X(t) e^{-k \Delta t}+\theta\left(1-e^{-k \Delta t}\right) \\
S(t)+v \Delta t
\end{array}\right], \\
\Sigma=\left[\begin{array}{cc}
\frac{\eta^{2}}{2 k}\left(1-e^{-2 k \Delta t}\right) & \frac{\rho \eta \sigma}{k}\left(1-e^{k \Delta t}\right) \\
\frac{\rho \eta \sigma}{k}\left(1-e^{k \Delta t}\right) & \sigma^{2} \Delta t
\end{array}\right] .
\end{gathered}
$$

derived from (34) and (35).

By taking the partial derivatives of the log of (38) (known as the log-likelihood function) with respect to each of the six parameters and setting the derivatives to zero, six equations with six unknowns are obtained. Solving the six simultaneous equations yield the following estimators (the details are not provided due to space considerations)

$$
\begin{aligned}
\hat{\sigma} & =\sqrt{\frac{\hat{S}^{2}}{\Delta t}}, \\
\hat{\mu} & =\frac{\hat{m}}{\Delta t}+\frac{1}{2} \hat{\sigma}^{2} \\
\hat{k} & =-\frac{\log (\hat{p})}{\Delta t} \\
\hat{\theta} & =\frac{\hat{q}}{1-\hat{p}}, \\
\hat{\eta} & =\sqrt{\frac{2 \hat{k} \hat{V}^{2}}{1-\hat{p}^{2}}} \\
\hat{\rho} & =\frac{\hat{k} \hat{C} \hat{V} \hat{S}}{\hat{\eta} \hat{\sigma}(1-\hat{p})},
\end{aligned}
$$

where

$$
\begin{aligned}
& \hat{m}=\frac{S(N)-S(0)}{N}, \\
& \hat{S}^{2}=\frac{\sum_{t=0}^{N-1}(S(t+1)-S(t))^{2}-2 \hat{m}(S(N)-S(0))+N \hat{m}^{2}}{N}, \\
& \hat{p}=\frac{1}{N \sum_{t=0}^{N-1} X(t)^{2}-\left(\sum_{t=0}^{N-1} X(t)\right)^{2}} \cdot\left[N \sum_{t=0}^{N-1}(X(t+1) X(t))\right. \\
& \left.-(X(N)-X(0)) \sum_{t=0}^{N-1} X(t)-\left(\sum_{t=0}^{N-1} X(t)\right)^{2}\right], \\
& \hat{q}=\frac{X(N)-x(0)+\sum_{t=0}^{N-1} X(t)-\hat{p} \sum_{t=0}^{N-1} X(t)}{N}, \\
& \hat{V}^{2}=\frac{1}{N}\left[X^{2}(N)-X^{2}(0)+\left(1+\hat{p}^{2}\right) \sum_{t=0}^{N-1} X^{2}(t)\right. \\
& \left.-2 \hat{p} \sum_{t=0}^{N-1} X(t) X(t+1)-N \hat{q}\right] \\
& \hat{C}=\frac{1}{N \hat{V} \hat{S}} \cdot\left[\sum_{t=0}^{N-1} X(t+1)(S(t+1)-S(t))\right. \\
& -\hat{p} \sum_{t=0}^{N-1} X(t)(S(t+1)-S(t)) \\
& -\hat{m}(X(N)-X(0)) \\
& \left.-\hat{m}(1-\hat{p}) \sum_{t=0}^{N-1} X(t)\right] \text {. }
\end{aligned}
$$

Thus, we obtain closed form expressions for the maximum likelihood estimators of the required parameter values. 